\title{
Genética y fútbol: asociación de los polimorfismos genéticos ACTN3 y ACE-I/D en jugadores de fútbol: Revisión literaria \\ Genetic and soccer: association of ACTN3 and ACE-I/D genetic polymorphisms in soccer players: Literary review \\ Wilson Arroyo Moya \\ Universidad de Ciencias Aplicadas y Ambientales (Colombia)
}

Resumen. Se ha comprobado y establecido que alrededor del $66 \%$ de los componentes del rendimiento y estado del atleta se explican por los factores hereditarios. Dos de los polimorfísmos genéticos mas estudiados con relación al rendimiento deportivo en la ultima década, son el ACTN3 R577X y el ACE I/D. El objetivo de este estudio fue resumir las posibles asociaciones de ambos polimorfismos y el rendimiento en jugadores de fútbol, determinando la combinación alélica y genotípica que más destaca en esta población y, además, observar las relaciones de estos a nivel físico y fisiológico. Para la realización de este estudio se llevaron a cabo dos fases, una primera de revisión bibliográfica en bases de datos (Pubmed, Scopus y Sportdiscus) y una segunda fase de clasificación y análisis de la información. Los estudios evidencian una mayor influencia de los alelos ACE-D y ACTN-R, y de los genotipos ACE-ID y ACTN-RR. Se observó que los sujetos con estas combinaciones tenían mejores rendimientos en las pruebas de velocidad y fuerza. Por otro lado, el polimorfismo ACTN3 está directamente relacionado con las lesiones musculares. En conclusión, se pudo observar una relación entre los polimorfismos genéticos y el rendimiento en jugadores de fútbol. Los componentes genéticos se pueden integrar como un nuevo componente dentro de la caracterización del deporte y como una herramienta dentro de un modelo de identificacción y detección de talentos en el fútbol juvenil. Además, los biomarcadores genéticos podrían ser responsables en un futuro, del estudio del riesgo de lesiones para que se optimice mucho más el rendimiento en el fútbol profesional.

Palabras clave: ACE I/D, ACTN3 R577X, genética deportiva, rendimiento, polimorfismo.

\begin{abstract}
It has been verified and established that around $66 \%$ of the components of the athlete's performance and state are explained by hereditary factors. Two of the most studied genetic polymorphisms in relation to sports performance in the last decade are ACTN3 R577X and ACE I/D. The aim of this study was to summarize the possible associations of both polymorphisms and the performance in soccer players, determining the allelic and genotypic combination that stands out the most in this population and, also, to observe their relationships at the physical and physiological level. In order to complete this study, two phases were carried out, the first one of literature review in databases (Pubmed, Scopus and Sportdiscus) and, the second one which covered the information classification and analysis. The studies show a greater influence of the ACE-D and ACTN-R alleles, and higher presence of the ACE-ID and ACTN-RR genotypes. Subjects with these combinations were found to perform better on speed and strength tests. On the other hand, the ACTN3 polymorphism is directly related to muscle injuries. In conclusion, a relationship between genetic polymorphisms and performance in soccer players could be observed. Genetic components can be integrated as a new component within the characterization of sport and as a tool within a model of identification and detection of talents in youth soccer. In addition, genetic biomarkers could be responsible, in the future, for the study of the risk of injury so that performance in professional soccer is much more optimized.
\end{abstract}

Key words: ACE I/D, ACTN3 R577X, sports genetics, performance, polymorphism.

\section{Introducción}

La terminación exitosa del proyecto genoma humano representó un avance histórico en la ciencia y en la vida del ser humano, motivando el uso de la genética en la solución de ecuaciones no tan claras, de aquellos componentes que nos hacen especiales. La genética ha empezado a jugar un papel protagónico (al igual que en otros campos) dentro de las ciencias del deporte, como un instrumento de ayuda (aún en desarrollo) en el proceso de identificación y selección de los talentos deportivos. Los primeros marcadores genéticos asociados con el rendimiento deportivo datan de principios del siglo XXI. Existe evidencia de 170 variantes genéticas, 17 marcadores de ADN mitocondrial y 25 marcadores genéticos nucleares en el mapa genético humano, que están relacionados con los fenotipos del rendimiento físico (Sgourou, Fotopoulos, Kontos, Patrinos, \& Papachatzopoulou, 2012). Además, se ha comprobado y establecido que alrededor del $66 \%$ de los componentes del rendimiento y estado del atleta se explican por los factores

Fecha recepción: 19-04-19. Fecha de aceptación: 18-07-20

Wilson Arroyo Moya

warroyo@udca.edu.co hereditarios (De Moor et al., 2007; Egorova et al., 2014). Por otro lado, Ahmetov y Fedotovskaya (2015), en una revisión sistematica, hablan de por lo menos 120 marcadores geneticos asociados con el estado del atleta de elite ( 77 marcadores relacionados con la resistencia y 43 con la fuerza/potencia). Dos de los polimorfismos genéticos mas estudiados con relación al rendimiento deportivo en la ultima decada, son el ACTN3 R577X y el ACE I/D.

ElACTN3 R577X es el gen que codifica la proteina alfaactinina-3, conocida como una de las proteinas mas importantes y funcionales de las lineas $\mathrm{Z}$ del sarcomero muscular. Dicha proteina esta ubicada en las fibras de contracción rápida tipo $2 \mathrm{~b}$ y sirve de ayuda en la estructura muscular, siendo el punto de anclaje de los filamentos de actina de los sarcomeros adyacentes, contribuyendo en la ejecución de contracciones musculares potentes y a la transmisión de la fuerza producida por dichas contracciones (Mills et al., 2001; Santiago et al., 2010). Este gen presenta tres combinaciones genotípicas (XX, RX y RR), de las cuales dos, expresan a la alfa-actinina-3 (RX y RR) y se han relacionado con disciplinas en las que la fuerza, velocidad $y$ potencia son capacidades predominantes, mientras que el genotipo que no expresa la proteína $(\mathrm{XX})$ ha sido relacionado con actividades de resistencia aeróbica (Coelho et al., 2018). 
Por otro lado, el polimorfismo ACE I/D se define por la presencia (inserción, alelo I) o la ausencia (deleción, alelo D) de un fragmento de $287 \mathrm{pb}$ (NHGRI, s.f), y del cual se derivan tres variantes genotípicas (II, ID y DD). El principal efecto de este gen, se da en el sistema renina-angiotensina-aldosterona, debido a que, con su activación, la angiotensina I se convierte en angiotensina II (conocida por su potente acción vasoconstrictora) e inactivando a la bradiquinina (conocida por su potencia vasodilatadora) (Moya, Madrigal, \& Zalasar, 2012), por ende, el aumento de la actividad de ACE I/D impulsa respuestas hipertensivas y disminuye las respuestas hipotensoras, desempeñando así, un papel crucial en la regulación de la presión arterial humana (Puthucheary, Skipworth, Rawal, Loosemore, Van Someren \& Montgomery, 2011). El alelo I ha sido asociado con una menor actividad enzimática del gen y ha sido altamente relacionado al rendimiento en deportes de resistencia, mientras que el alelo D se asocia con una mayor actividad enzimática, aumentando los niveles de angiotensina II y relacionándolo con el rendimiento en deportes de fuerza/potencia/velocidad (Jones, Montgomery \& Woods, 2002; Eider, Cieszczyk, Ficek, Leonska-Duniec, Sawczuk, Maciejewska-Karlowska \& Zarebska, 2013).

El fútbol, al igual que otros deportes, ha sido objeto de estudio para determinar la relación de ambos polimorfismos y el rendimiento en jugadores de fútbol profesional y amateur. El objetivo de este estudio es resumir las posibles asociaciones de ambos polimorfismos y el rendimiento en jugadores de fútbol, determinando la combinación alélica y genotípica que más destaca en esta población y, además, observar las relaciones de estos a nivel físico y fisiológico.

Conceptos básicos: genética, polimorfismo, epigenética, genómica, genotipo, fenotipo y alelo

Es conveniente conceptualizar, de manera breve, algunos términos importantes que enmarcan la temática de este estudio.

En primer lugar, la genética es considerada como la ciencia que estudia las formas en que se hereda la información contenida en los genes de una generación a otra, es decir, la transmisión de rasgos y fenotipos a través de generaciones (Copelli, 2010, p.6; Bouchard, Rankinen \& Timmons, 2011). En referencia a los polimorfismos genéticos, se ha comprobado que el $99,9 \%$ de la secuencia de $\mathrm{ADN}$ en dos sujetos diferentes es análoga y que el 0,1\% restante, radica en las incompatibilidades fenotípicas (color de ojos o de piel, tipo de cabello o funciones fisiológicas) y/o susceptibilidad a ciertas enfermedades, este pequeño porcentaje es al que se denomina como polimorfismo genético (Caratachea, 2007). Entonces, ¿Cómo puede ser que tener solo una pequeña variación genética cree una gran diferencia? La respuesta la tiene la epigenética. Esta describe los cambios heredables en la expresión génica que ocurren en ausencia de cambios en la secuencia de ADN (Ehlert, Simon, \& Moser, 2013). Las modificaciones epigenéticas son la metilación del ADN y las modificaciones de histonas (García-Robles, Ayala-Ramírez, \& Perdomo-Velásquez, 2012). Dichos cambios en la expresión génica se deben principalmente a efectos relacionados con el medio externo y estímulos como el entrenamiento.

Por otro lado, la genómica es el conjunto de técnicas y ciencias que se dedican al estudio integral de los genomas, se encarga de comprender su funcionamiento, su evolución y el origen. Aunque conceptualmente son diferentes, la genética y la genómica comparten las unidades de estudio principales: el ADN y los genes (Sánchez, Campuzano, Iglesias, \& Brugada, 2009). En cuanto al genotipo y el fenotipo, el primero representa todo el material genético de un individuo, mientras que el segundo, es cualquier característica o rasgo visible de un organismo, como su desarrollo, propiedades bioquímicas o su fisiología y conducta (Muniesa, Santiago, Gómez-Gallego, \& Lucía, 2011) Por último, un alelo es «cada una de las dos o más versiones de un gen. Un individuo hereda dos alelos para cada gen, uno del padre y el otro de la madre. Los alelos se encuentran en la misma posición dentro de los cromosomas homólogos. Si los dos alelos son idénticos, el individuo es homocigoto para este gen. En cambio, si los alelos son diferentes, el individuo es heterocigoto para este gen» (NHGRI, s.f).

\section{Materiales y métodos}

\section{Diseño de investigación}

Este es un estudio de tipo descriptivo propositivo y para su realización, se llevaron a cabo dos fases, una primera de revisión bibliográfica y una segunda fase de clasificación y análisis de la información.

Fase 1. Revisión bibliográfica: Se realizó una búsqueda detallada en las bases de datos PubMed, Scopus y Sportdiscus. Se usaron los títulos de términos médicos (Medical Subjects Headings) MESH y la ecuación de búsqueda «ACE I/D» and «ACTN R577X or «polymorphism» $\mathrm{y}$ «soccer players». La búsqueda bibliográfica se limitó a los estudios desde el 1 de enero de 2013 hasta el 31 de diciembre de 2019, ya que este marco de tiempo permite capturar investigaciones más recientes. Los criterios de inclusión de los artículos fueron: Artículos donde los polimorfismos ACE I/D y ACTN R577X fueran objeto de estudio, estudios donde participaran deportistas profesionales o amateurs, se consideró cualquier tipo de diseño investigativo, solo se incluyeron artículos en inglés y publicados en revistas percentil Q1, Q2 y Q3 según la plataforma Scimago. Se excluyeron trabajos no publicados, actas de conferencias y resúmenes.

Fase 2. Clasificación de la información: Los artículos fueron clasificados de acuerdo a las siguientes temáticas: Frecuencias del polimorfismo en los jugadores de fútbol, polimorfismos y rendimiento en test físicos e influencias fisiológicas del polimorfismo.

\section{Resultados}

La búsqueda inicial arrojo un total de cuatrocientos veintinueve (429) artículos que podrían ser potencialmente buenos para este estudio. Posteriormente se excluyeron artículos con base al título, al resumen y eliminación de los artículos duplicados. Todo se redujo a un total de trece artículos potencialmente buenos para este estudio. Según la clasificación por temáticas la información quedo distribuida de la siguiente manera: Frecuencia del polimorfismo en los jugadores de fútbol (siete artículos), polimorfismo y 
rendimiento en test físicos (tres artículos), y las influencias fisiológicas del polimorfismo (tres artículos) (Tabla 1). Los trece estudios incluidos en esta revisión, utilizaron dentro de sus muestras deportistas de fútbol profesional. Los tamaños de las muestras de los jugadores profesionales variaron en todos los estudios, desde $n=25$ hasta $n=246$.

Frecuencia del polimorfismo en los jugadores de fútbol

Para la primera temática de estudio, se encontraron siete artículos que indicaron las frecuencias de ambos polimorfismos en los grupos de deportistas. En cuanto a las frecuencias del ACE I/D, tres estudios reportaron mayor frecuencia para el genotipo ID y los otros dos estudios para el genotipo DD, ningún estudio reporto una mayor frecuencia para el genotipo II (figura 1). Todos los estudios hallaron una presencia muy alta del alelo D en comparación con el alelo I (figura 2) y, además, indicaron una alta relación del polimorfismo con el rendimiento de los jugadores.
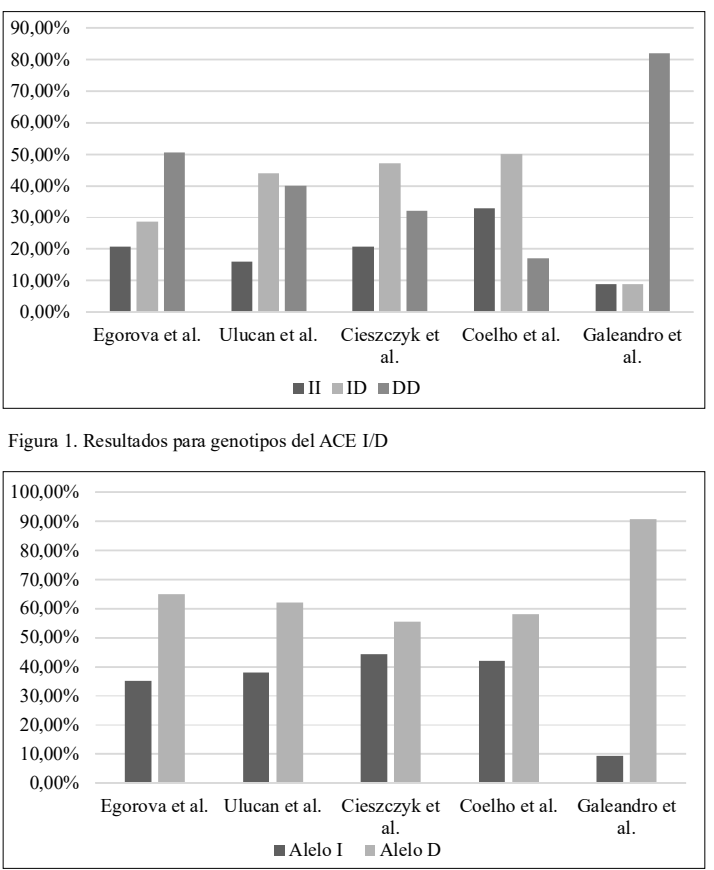

Figura 2. Resultados para alelos del ACE I/D

Por otro lado, los resultados obtenidos para el polimorfismo ACTN3, indican una mayor frecuencia del genotipo RR en cuatro de los cinco estudios encontrados, el genotipo RX fue el de mayor frecuencia en el estudio de Coelho et al. (2018 a) (figura 3). El alelo R (vs alelo X), fue muy superior en todos los estudios (figura 4).

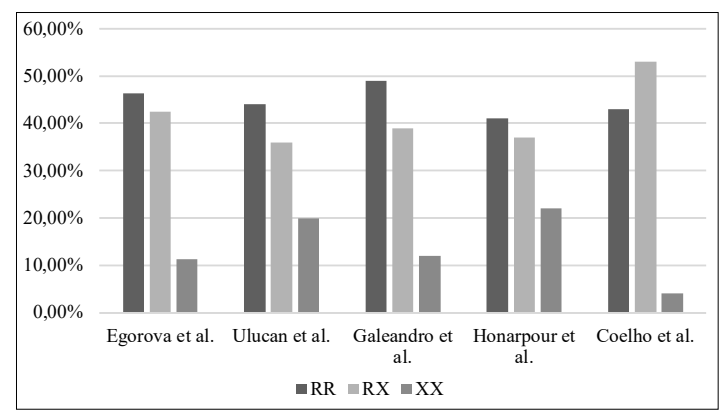

Figura 3. Resultados para genotipos del ACTN3

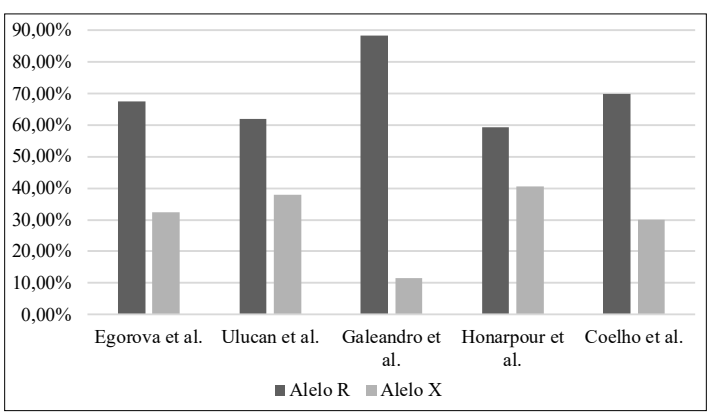

Figura 4. Resultados para alelos del ACTN3

\section{Polimorfismos y rendimiento en test físicos}

Para esta temática se encontraron tres estudios potencialmente buenos que compararon la relación de los polimorfismos y el rendimiento físico de los jugadores, a través de la aplicación de tests de campo.

Los estudios de Pimienta et al. (2013) y Coelho et al. (2016 b), tuvieron similitudes en los tests de campo aplicados a los grupos de estudio. Los test escogidos, median tres capacidades físicas fundamentales, velocidad $(\mathbf{3 0} \mathbf{~ m})$, fuerza (Salto contra movimiento $[\mathbf{C M J}]$ y test de Bosco o Squat jump [SJ]) y resistencia aeróbica (Yo-Yo test). El estudio de Pimienta et al. (2013), tuvo como polimorfismo de estudio el ACTN3 en una muestra de $n=200$ jugadores de primera división de fútbol de Brasil que fueron separados en tres grupos dependiendo de los resultados para las frecuencias genotípicas del polimorfismo $(\mathrm{RR}=45 \%, \mathrm{RX}=44 \%, \mathrm{XX}=11 \%)$. Sus resultados indicaron que el grupo $\mathrm{XX}$ obtuvo un rendimiento mucho mayor en la prueba del Yo-Yo test, en comparación con los otros dos grupos. Para el test de $30 \mathrm{~m}$, los individuos del grupo RR presentaron tasas de tiempo más bajas que los grupos RX y XX. En cuanto a los tests de salto que median la fuerza de tren inferior, los individuos de los grupos RR y RX registraron mejores saltos que los individuos XX.

Por otro lado, el estudio de Coelho et al. (2016 b) estudio el mismo gen, pero en una población de $n=138$ futbolistas y reportaron los siguientes resultados: Para los test de velocidad los individuos con 
genotipo RR fueron superiores a los otros dos grupos; el test de resistencia logró una paridad para los grupos RR y $\mathrm{RX}$, resultado que fue similar para los test de salto para fuerza de tren inferior. En este estudio se relacionaron las frecuencias alélicas del polimorfismo y todos los individuos de alelo dominante $\mathrm{R}$ fueron superiores en todas las pruebas. Por último, el estudio de Dionisio et al. (2017), investigo si los polimorfismos ACTN3 y ACE I/D afectaban las pruebas de fuerza, velocidad y resistencia en $n=220$ jugadores del equipo de Sao Pablo (Brasil). Los principales resultados del estudio fueron que los deportistas que portaban genotipos RR, RX y DD presentaron mejor rendimiento durante los test de fuerza y velocidad, y los deportistas con genotipos ID/II presentaron mejores resultados durante el test de resistencia.

\section{Influencias fisiológicas del polimorfismo}

Para esta temática fueron encontrados tres artículos científicos, todos en relación al gen ACTN3 y su influencia en factores fisiológicos.

Massida et al. (2017) estudiaron la asociación del polimorfismo y las tasas de lesiones en $n=169$ futbolistas (subgrupo de estudio) de Italia durante 5 temporadas (20052014). Dentro de sus hallazgos revelaron que el genotipo XX (deficiencia de alfa-actinina-3) tiene una mayor incidencia y gravedad en las lesiones de los jugadores de fútbol profesional; los futbolistas que poseían este genotipo tenían $2.66 \%$ de probabilidades más altas de incidencia de lesiones y $2.13 \%$ de probabilidades más altas de lesiones graves en comparación con sus homólogos RR.

De igual manera, Clos et al. (2019) estudiaron un total de $\mathrm{n}=43$ futbolistas profesionales, en donde recopilaron datos de lesiones sufridas en siete temporadas diferentes (20072012 y 2015-2017). En sus resultados, los jugadores que poseían genotipo XX tuvieron la tasa de lesiones más alta (2.78), seguidos por el grupo RR (1.51) y RX (0.83), respectivamente. Por último, Coelho et al. (2018) evaluaron los indicadores de daño muscular (Creatin-kinasa [CK] y alfaactina) y las respuestas hormonales (Interleucina-6, cortisol y testosterona) antes y después de los partidos de fútbol y su relación con la expresión del polimorfismo ACTN3 en $\mathrm{n}=30$ futbolistas. La evaluación fue llevada a cabo antes, inmediatamente después, dos y cuatro horas después de la competencia. Sus resultados indicaron que no hubo una diferencia significativa entre los genotipos, aunque fue un poco mayor en individuos XX.

\section{Discusión}

Las demandas fisiológicas durante un partido de fútbol han sido estudiadas por varios autores (Bangsbo, 1994; Reillly, Bangsbo \& Franks, 2000; Stølen, Chamari, Castagna \& Wisløff, 2005). La mayor parte de la actividad durante un juego de fútbol se caracteriza por ser «off the ball» o acciones sin balón, que son acciones principalmente aeróbicas, mientras que las actividades que están directamente involucradas en el juego con balón, son en gran medida de requerimiento anaeróbico. De hecho, las acciones de vía anaeróbica pueden constituir los momentos más cruciales del juego (Reilly, Bangsbo, \& Franks, 2000). Por ejemplo, Stolen et al. (2005) describen que, en un partido de fútbol, un jugador profesional puede llegar a realizar entre 10-20 sprints, carreras de alta intensidad aproximadamente cada 70 segundos, alrededor de 15 entradas, 10 cabezazos, 50 desarrollos con balón, aproximadamente 30 pases, así como cambios de ritmo y contracciones decisivas para mantener el equilibrio y el control del balón en jugadas con presión defensiva. A partir de esto, y con la genómica deportiva en desarrollo, se han permitido establecer las características genéticas que pueden llegar a ser fundamentales dentro de un deporte como el fútbol, por ende, el propósito de este estudio de observar las asociaciones de los polimorfismos genéticos ACTN3 y ACE I/D con el rendimiento en jugadores de fútbol. El fin de observar las relaciones de estos a nivel físico y fisiológico, representa una herramienta nueva dentro de la caracterización del deporte.

El alelo D ha sido asociado con una mayor actividad circulante de la ACE, siendo correlacionada significativamente con un mayor volumen y fuerza muscular, al igual que con un mayor porcentaje de fibras musculares de contracción rápida, factor de crecimiento e hipertrofia (Puthucheary et al., 2011; Alvarez, 2000). Este alelo ha sido relacionado con el rendimiento en atletas de potencia Polacos (Eider et al., 2013), nadadores de distancias cortas (Costa et al., 2009), entre otros (Woods et al., 2001; Jones et al., 2002). Los resultados para las frecuencias alélicas, indican que el alelo D fue el de mayor frecuencia en todos los estudios. Por otro lado, el polimorfismo ACTN3 ha sido potencialmente relacionado con el rendimiento deportivo y en algunas investigaciones el alelo $\mathrm{R}$ relacionado con deportes de fuerza y potencia (Yang et al., 2003; Papadimitriou et al., 2008). En esta revisión, el alelo R fue el de mayor actividad en todos los estudios. Teniendo en cuenta que el fútbol está clasificado como una actividad intermitente y muy intensa, y que el rendimiento de los jugadores está directamente influenciado por la velocidad, la potencia y la capacidad de fuerza de los jugadores (Coelho et al., 2016 a), son entendibles los resultados de los estudios para las frecuencias alélicas.

En cuanto a los resultados para las frecuencias genotípicas, se encontró una mayor frecuencia del genotipo ID, seguido por el DD y una menor frecuencia del genotipo II dentro de los estudios. El hecho de que el genotipo ID fuera el de mayor actividad, sugiere y soporta, según los estudios, que las características del fútbol, están determinadas por un perfil metabólico mixto (aeróbico/anaeróbico) (Coelho et al., 2016 a). Por otro lado, los resultados para el polimorfismo ACTN3, presentan al genotipo RR como el más influyente, seguido por el RX y, por último, el genotipo XX. Teniendo en cuenta las características actuales del fútbol, en donde los resultados están casi siempre determinados por la fuerza y la velocidad, y en donde crecen cada vez las demandas físicas del entrenamiento y la competencia, una menor frecuencia para los genotipos ACE-II y ACTN3-XX, y para los alelos I y $\mathrm{X}$, representan coherentemente el perfil genético de estos deportistas.

Para facilitar el entendimiento de los resultados anteriores, fue pertinente observar estudios en donde se usarán y compararán los resultados de herramientas de evaluación para las capacidades físicas como la velocidad, la resistencia, la fuerza y los polimorfismos en contexto.

Para el caso del polimorfismo ACTN3, los resultados 
indican que los futbolistas con combinación $\mathrm{RR} / \mathrm{RX}$ tienen mejores rendimientos en pruebas de fuerza/velocidad y que los sujetos con genotipo XX se destacan en pruebas de resistencia. Una explicación fisiológica para estos resultados radica en el fenotipo estructural del musculo (Coelho et al., 2016 b), en donde el ACTN3 tiene relación directa con la línea $\mathrm{Z}$ muscular, que cumple la función de mantener la integridad del aparato contráctil durante el esfuerzo muscular intenso en fibras musculares tipo II (Coelho et al., 2016 b). El tener deficiencia de ACTN3 (alfa-actinina-3) conlleva, muy probablemente, ha cambios funcionales del musculo. Según los estudios, esta deficiencia aumenta la participación de otras proteínas, principalmente actn2 (a-actinina-2) y titina, que son determinantes de la rigidez y tensión pasiva del sarcómero, particularmente en los músculos de contracción rápida, dando como resultado, que las propiedades elásticas de los mismos sean insuficientes (Seto et al., 2011). Por lo tanto, estos cambios estructurales, podrían explicar, una parte de la reducción del rendimiento en futbolistas o atletas con genotipo XX que realizan actividades en las que los componentes elásticos del ciclo estiramiento-acortamiento están presentes, como el sprint y el salto (Pimienta et al., 2013). Por ende, se esperaría que los futbolistas RR/RX, que expresan la alfa-actina-3, muestren una mayor adaptación al entrenamiento pliométrico (potencia) y que resalten en actividades que requieren este tipo de contracción muscular (Dionisío et al., 2017).

En cuanto al polimorfismo ACE, los resultados indicaron que los futbolistas con genotipo DD fueron mejores en las pruebas de salto y velocidad, mientras que los jugadores con genotipos ID/II obtuvieron mejores resultados en el test de resistencia. Como ya se mencionó antes, el alelo D ha sido asociado con una mayor producción de ACE circulante y local, aumentando los niveles de angiotensina II (Jones et al., 2002; Eider et al., 2013). Respecto a esto, en un estudio llevado a cabo por Rattigan et al. (1996) citado en Dionisío et al. (2017), se observó que la angiotensina II puede ser crucial en la circulación muscular, debido a que direcciona el flujo sanguíneo de las fibras musculares tipo I a las fibras musculares tipo II, mejorando sustancialmente el rendimiento en actividades de potencia. Este mismo estudio resalta que, la angiotensina II aumenta la absorción de oxigeno por parte del musculo, es decir, una mayor producción de angiotensina II facilitaría la contracción muscular potente y perjudicaría las acciones aeróbicas.

Por último, fue pertinente observar las posibles relaciones fisiológicas que pudieran afectar el rendimiento deportivo de los jugadores. Dentro de esta temática, todos los estudios encontrados estuvieron relacionados con el polimorfismo ACTN3 y su relación directa con las lesiones y trastornos musculares en los futbolistas.

En los estudios de Massida et al. (2017) y Clos et al. (2019), se observó que los jugadores con genotipo XX tienen mayor incidencia de sufrir lesiones musculares $(2.66 \%$ más que los de genotipo RR) y una tasa de lesiones más alta, que los otros dos genotipos $(\mathrm{XX}=2.78, \mathrm{RR}=1.51, \mathrm{RX}=0.83)$. Según Massida et al. (2017), la base fisiológica para esta condición de susceptibilidad al daño muscular, se atribuye generalmente a cambios en las estructuras de transmisión de la fuerza generacional de los miofilamentos, pero en particular, a los cambios en las proteínas del cito esqueleto a nivel del disco Z. Más específicamente, mientras que los sujetos RX y RR tienen una buena presencia de a-actininina-3, los sujetos $\mathrm{XX}$ no, quienes se compensan, como anteriormente se mencionó, con la isoforma de actn2 y tinina. Aunque una deficiencia de a-actinina-3 no produce una alteración manifiesta en la estructura de los sarcómeros, probablemente por la regulación compensatoria de la actina-2, su ausencia produce diferencias en la función del músculo esquelético, incluida una menor fuerza muscular (Seto et al., 2011). Entonces, la ausencia de á-actinina-3 (genotipo XX) parece estar asociada a sufrir más daño muscular por la actividad física (Coelho et al., 2018). Por otro lado, Clos et al. (2019) indicaron que los sujetos con genotipo XX, aunque tienen una incidencia más alta de lesiones, requieren menos tiempo de recuperación. Por otro lado, la presencia de á-actinina-3, que es mayor en el genotipo RR que en el genotipo RX, parece estar asociada con tener menor daño muscular pero una recuperación más lenta (Lee, Houweling, North, \& Quinlan, 2016). En resumen, la alfa-actinina, además de desempeñar un papel mecánico, actúa como un sensor metabólico y perjudicaría las respuestas musculares en contracciones excéntricas o movimientos de alta potencia en individuos con genotipo XX, lo que causaría mayor tensión en estas fibras y, en consecuencia, más catabolismo (Coelho etal., 2019).

Finalmente, Coelho et al. (2019) demostraron, en un grupo de 30 jugadores de fútbol, que los individuos con genotipo XX tuvieron concentraciones más altas de marcadores de micro trauma muscular (CK y alfa-actina), esto se observó en todos los puntos de tiempo excepto a las cuatro horas después del juego. Aunque informaron que no hay diferencia en la predisposición hacia el micro trauma muscular entre los diferentes genotipos de ACTN3, porque no hay diferencias en las concentraciones plasmáticas de $\mathrm{CK}$, sugieren que los individuos XX son más susceptibles al daño muscular. Por ejemplo, Pimienta et al. (2012) en un estudio realizado en 37 jugadores de futbol profesional, hallaron que los jugadores con genotipo XX tenían niveles elevados de marcadores de daño muscular [CK (cuatro horas después), a-actina (después y dos horas después), y cortisol (post)] después de un entrenamiento excéntrico, en comparación a los jugadores con genotipos RX y RR.

En cuanto a las respuestas hormonales, los valores de interleucina-6(IL-6) fueron casi el doble para el grupo XX en comparación con el grupo RR/RX antes y después del juego. Cabe resaltar que la IL-6 juega un papel importante como antiinflamatorio, pero que su principal objetivo es el de mantener los sustratos energéticos para que se pueda continuar una actividad (Fischer, 2006). Su producción se relaciona directamente con la señalización intracelular de calcio, es decir que, en actividades de larga duración, la necesidad de mantener una disponibilidad de calcio crea las condiciones perfectas para la producción de IL-6 (Coelho et al., 2019). En resumen, este perfil es típico para las actividades aeróbicas, fibras tipo I y para los sujetos con genotipo XX. Respecto a la testosterona, los individuos con genotipo RR/ RX tuvieron concentraciones más altas inmediatamente después del juego y hasta cuatro horas más tarde que antes del juego. La explicación para esto es que las actividades de 
alta intensidad (sabiendo ya, que el fútbol es un deporte que requiere de movimientos intensos) requieren una mayor producción de testosterona, debido al aumento en las concentraciones de epinefrina y lactato que influyen en la actividad gonadal (Canali \& Kruel, 2001; Derbré et al., 2010). Por último, el estudio revelo que antes del juego, el grupo $\mathrm{XX}$ tenía mayores concentraciones de cortisol que los genotipos RR/RX, lo que indica una situación más catabólica en este grupo. Teniendo en cuenta que la concentración de cortisol antes del juego corresponde a la condición física general de los jugadores, un estado de entrenamiento más catabólico puede ser la consecuencia de una menor recuperación de la carga de entrenamiento (Coelho et al., 2019).

Estos hallazgos son importantes y novedosos para la caracterización de un deporte como el fútbol, en donde las variables, dimensiones e indicadores de rendimiento más evaluados hasta la actualidad han sido físicas, fisiológicas, psicológicas, técnico-tácticas y entre otras (Serra-Olivares, Prieto-Ayuso, Pastor-Vicedo, \& González-Víllora, 2020). Es importante mencionar que la estructura genética del ser humano no solo determina el color de piel, ojos o del cabello de las personas, sino también, cada uno de los procesos metabólicos que se llevan a cabo dentro del cuerpo (ArroyoMoya, Rodríguez-Buitrago, \& Escarria-Moreno, 2020) y cuando se observan dichas predisposiciones, se llega a entender que la edad, el género, las características anatómicas y fisiológicas, el sistema nervioso, toda la estructura cardiovascular y las habilidades biomotoras básicas, dependen del componente genético de cada individuo, lo cual determinaría el rendimiento deportivo de los sujetos (Ozveren, Ozcaldiran, Durmaz, \& Oral, 2014; Arroyo-Moya, Rodríguez-Buitrago, \& Escarria-Moreno, 2020). De igual manera, otras características tales como la automotivación, la disciplina, así como la baja susceptibilidad a las lesiones, tienen todos componentes genéticos y epigenéticos (Ehlert, Simon, \& Moser, 2013).

Por otro lado, se ha descrito que variables individuales como el estado socioeconómico, la nutrición, el manejo del estrés psicológico y muchos otros, tienen correlación genética y epigenética que pueden contribuir a resultados negativos o positivos en estudios de asociación que buscan el «genotipo atlético perfecto» (Ehlert, Simon, \& Moser, 2013). Es importante resaltar que se sabe poco sobre las posibles influencias epigenéticas en el rendimiento físico en humanos. Sin embargo, los primeros estudios han demostrado un claro impacto de las marcas epigenéticas en las vías fisiológicas, incluidos los rasgos de alta relevancia para la actividad locomotora y la aptitud física (Ehlert, Simon, \& Moser, 2013).

El hecho de que el fenotipo de una persona esté determinado por el genotipo y el ambiente, resalta el grado de influencia de cada uno de estos factores en las cualidades físicas y, más en particular, en el rendimiento deportivo (Padullés i Riu, Terrados, Rodas, \& Campos, 2004). Sin embargo, la investigación sobre genética y epigenética en la prescripción del entrenamiento es limitada (Suraci, Quigley, Thelwell, \& Milligan, 2019). Con relación a esto último, Kikuchi y Nakazato (2015) plantean que los entrenadores de clubes de fútbol profesional pueden mejorar el rendimiento deportivo de cada atleta, si adaptan programas específicos de acondicionamiento físico, dentro del principio de individualización, que estén basados en la composición genética de cada atleta. Teniendo en cuenta que los jugadores de fútbol profesional ya tienen una buena formación y están en su extremo de rendimiento, parece apropiado pensar que el contenido de entrenamiento físico debe ser realizado de manera individualizada (Pimienta et al., 2013). Es decir, los jugadores RR/RX y DD/ID deberían realizar un entrenamiento de velocidad y potencia más alto que los grupos XX o II, en donde estos últimos realicen un entrenamiento aeróbico más alto (Pimienta et al., 2013).

\section{Conclusión y futuras líneas de investigación}

En conclusión, se pudo observar una relación entre los polimorfismos genéticos ACE I/D y ACTN3 y el rendimiento en jugadores de fútbol. Al observar los resultados, estos sugieren una relación y una combinación significativa entre los alelos ACE-D y ACTN-R, los genotipos ACE-ID y ACTNRR y las capacidades de velocidad/fuerza/potencia. Los jugadores con estás combinaciones, tuvieron mejor desempeño en las pruebas de velocidad y fuerza. Es evidente que estás investigaciones nos dan un nuevo componente para la caracterización del deporte, $\mathrm{y}$, además, aunque se necesitan más estudios en este campo, sugerir la utilización de los estudios de componentes geneticos, como una herramienta dentro de un modelo de identificacción y detección de talentos en el fútbol. Por otro lado, los biomarcadores genéticos podrían ser responsables en un futuro próximo del estudio del riesgo de lesiones y de que la evaluación de las lesiones se individualice mejor y se optimice el rendimiento en el fútbol profesional. Además, la aplicación de pruebas genéticas en los deportes podría proporcionar nuevas oportunidades para que los clubes deportivos entiendan la susceptibilidad de los atletas a ciertos estados patológicos (cardiomiopatía, muerte súbita, etc.), mapeen la idoneidad genética para puestos y roles específicos del equipo, y para ganar ideas sobre el desarrollo de los atletas en diversos deportes o actividades físicas (Ahmetov \& Fedotovskaya, 2015). De igual manera podrían realizarse entrenamientos individualizados dependiendo de las características genéticas que posea el jugador.

En países como Colombia son escasas las investigaciones de este tipo. Realizarlas, sería un paso importante en el desarrollo deportivo y en futuras líneas de investigación, debido a que determinar las características genéticas de nuestros deportistas y para nuestro caso, los futbolistas, integra un factor importante en la caracterización deportiva y el desarrollo científico. Se sugiere realizar no solo investigaciones con los polimorfismos en cuestión, sino con otros genes que han sido objeto de estudio en el rendimiento deportivo.

\section{Referencias}

Ahmetov, I., \& Fedotovskaya, O. (2015). Current Progress in Sports Genomics. Advances in Clinical Chemistry, 70, 247-314. doi:10.1016/bs.acc.2015.03.003.

Alvarez, R., Terrado, N., Ortolano, R., Iglesia-Cubero, G., 
Reguero, J., Batalla, A., ... Coto, E. (2000). Genetic variation in the renin-angiotensin system and athletic performance. European Journal of Applied Physiology, 82(1-2), 117120. doi: $10.1007 / \mathrm{s} 004210050660$

Arroyo-Moya, W., Rodríguez-Buitrago, A., \& EscarriaMoreno, A. (2020). La nueva frontera de la preparación deportiva, la genética y el polimorfismo ACE I/D en atletas de resistencia. Revista Digital: Actividad Física y Deporte, 6(2), 100-117. doi:10.31910/rdafd.v6.n2.2020.1571

Bangsbo, J. (1994). Energy demands in competitive soccer. Journal of Sports Sciences, 12, S5- S12. doi:https:// doi.org/10.1080/02640414.1994.12059272

Bouchard, C., Rankinen, T., \& Timmons, J. (2011). Genomics and Genetics in the Biology of Adaptation to Exercise. Comprehensive Physiology, 1(3), 1603-1648. doi:10.1002/ cphy.c100059

Canali, E., \& Kruel, L. (2001). Respostas hormonais ao exercício. Rev. paul. Educ. Fís., São Paulo 15 (2), 141153.

Caratachea, M. (2007). Polimorfismos genéticos: Importancia y aplicaciones. Revista del Instituto Nacional de Enfermedades Respiratorias, 20(3), 213-221.

Ciêszczyk, P., Leoñska-Duniec, A., Maciejewska-Skrendo, A., Sawczuk, M., LeŸnicka, K., Contrò, V., . . . Luliñska-Kuklik. (2016). Variation in the ACE gene in elite Polish football players. Human Movement, 17(4), 237-241. doi:https:// doi.org/10.1515/humo-2016-0032

Clos, E., Pruna, R., Lundblad, M., Artells, R., \& EsquirolCaussa, J. (2019). ACTN3 single nucleotide polymorphism is associated with non-contact musculoskeletal softtissue injury incidence in elite professional football players. Knee Surg Sports Traumatol Arthrosc, 27(12), 4055-4061. doi:10.1007/s00167-019-05381-x

Coelho, D., Pimenta, E., Cruz, I., Veneroso, C., Pussieldi, G., Becker, L., . . S Silami-Garcia, E. (2016 a). Angiotensinconverting enzyme (ACE-I/D) polymorphism frequency in Brazilian soccer players. Applied Physiology, Nutrition and Metabolism, 41(6), 692-694. doi:10.1139/apnm-20150514

Coelho, D., Pimenta, E., Rosse, I., de Castro, B., Becker, L., de Oliveira, E., .. . Garcia, E. (2018 ). Evidence for a Role of ACTN3 R577X Polymorphism in Football Player's Career Progression. International Journal of Sports Medicine, 39(14), 1088-1093. doi:10.1055/a-0753-4973

Coelho, D., Pimenta, E., Rosse, I., Veneroso, C., Pussieldi, G. Becker, L., .. . Silami-Garcia, E. (2019). Alpha-Actinin-3 R577X Polymorphism Influences Muscle Damage and Hormonal Responses After a Soccer Game. Journal of Strength \& Conditioning Research, 33(10), 2655-2664. doi:10.1519/JSC.0000000000002575

Coelho, D., Pimienta, E., Rosse, I., Veneroso, C., Becker, L., Carvalho, M., .. . Silami-Garcia, E. (2016 b). The alphaactinin-3 R577X polymorphism and physical performance in soccer players. Journal of Sports Medicine and Physical Fitness, 56(3), 241-248. Obtenido de https:// pubmed.ncbi.nlm.nih.gov/25650734/

Copelli, S. (2010). Genética : desde la herencia a la manipulación de los genes. Buenos Aires: Fundación de Historia Natural Félix de Azara.

Costa, A., Silva, A., Garrido, N., Louro, H., De Oliveira, R., \&
Breitenfeld, L. (2009). Association between ACE D allele and elite short distance swimming. European Journal of Applied Physiology, 106(6), 785-790. doi:10.1007/s00421009-1080-z

De Moor, M., Spector, T., Cherkas, L., Falchi, M., Hottenga, J., Boomsma, I., \& De Geus, E. (2007). Genome-wide linkage scan for athlete status in 700 British female DZ twin pairs. Twin Research and Human Genetics, 10(6), 812-820. doi:10.1375/twin.10.6.812

Derbré, Vincent, Maitel, Jacob, Delamarche, Delamarche, \& Zouhal. (2010). Androgen responses to sprint exercise in young men. International Journal of Sports Medicine, 31(5), 291-297. doi:10.1055/s-0030-1248243

Dionísio, T., Thiengo, C., Brozoski, D., Dionísio, E, Talamoni, G., . . Amaral, S. (2017). The influence of genetic polymorphisms on performance, cardiac and hemodynamic parameters among Brazilian soccer players. Applied Physiology, Nutrition and Metabolism, 42(6), 596-604. doi:https://doi.org/10.1139/apnm-2016-0608

Egorova, E., Borisova, A., Mustafina, L., Arkhipova, A., Gabbasov, R., Druzhevskaya, A., ... Ahmetov, I. (2014). The polygenic profile of Russian football players. Journal of Sports Sciences, 32(13), 1286-1293. doi:10.1080/ 02640414.2014.898853

Ehlert, T., Simon, P., \& Moser, D. (2013). Epigenetics in Sports. Sports Medicine, 43, 93-110. doi:10.1007/s40279-0120012-y

Eider, J., Cieszczyk, P., Ficek, K., Leonska-Duniec, A., Sawczuk, M., Maciejewska-Karlowska, A., \& Zarebska,A. (2013). The association between D allele of the ACE gene and power performance in Polish elite athletes. Science \& Sports, 28(6), 325-330. doi:10.1016/j.scispo.2012.11.005

Fischer, C. (2006). Interleukin-6 in acute exercise and training: What is the biological relevance? Exercise Immunology Review, 12, 6-33. Obtenido de https:// www.ncbi.nlm.nih.gov/pubmed/17201070

Galeandro, V., Notarnicola, A., Bianco, A., Tafuri, S., Russo, L., Pesce, V., . . Petruzzella, V. (2017). ACTN3/ACE genotypes and mitochondrial genome in professional soccer players performance. J Biol Regul Homeost Agents, 31(1), 207-213. Obtenido de https:// europepmc.org/article/med/28337894

García-Robles, R., Ayala-Ramírez, P., \& Perdomo-Velásquez, S. (2012). Epigenética: definición, bases moleculares e implicaciones en la salud y en la evolución humana. Revista Ciencia y Salud, 10(1), 59-71.

Honarpour, A., Mohseni, M., Hajiagha, S., Irani, S., \& Najmabadi. (2017). Investigation of the Relationship Between a Genetic Polymorphism in ACTN3 and Elite Sport Performance Among Iranian Soccer Players. Iranian Rehabilitation Journal, 15(2), 149-154. doi:10.18869/nrip.irj.15.2.149

Jones, A., Montgomery, H., \& Woods, D. (2002). Human Performance: A Role for the ACE Genotype? Exercise and Sport Sciences Reviews, 30(4), 184-190. Obtenido de https://journals.lww.comx

Kikuchi, N., \& Nakazato, K. (2015). Effective utilization of genetic information for athletes and coaches: focus on ACTN3 R577X polymorphism. Journal of Exercise Nutrition \& Biochemistry, 19(3), 157-164. doi:10.5717/ 
jenb.2015.15093001

Lee, F., Houweling, P., North, K., \& Quinlan, K. (2016). How does á-actinin-3 deficiency alter muscle function? Mechanistic insights into ACTN3, the 'gene for speed'. Biochimica et Biophysica Acta , 1863(4), 686-693. doi:10.1016/j.bbamcr.2016.01.013

Massidda, M., Voisin, S., Culigioni, C., Piras, F., Cugia, P., Yan, X., ... Calò, C. (2019). ACTN3 R577X Polymorphism Is Associated With the Incidence and Severity of Injuries in Professional Football Players. Clinical Journal of Sport Medicine, 29(1), 57-61. doi:10.1097/ JSM.0000000000000487

Mills, M., Yang, N., Weinberger, R., Vander-Woude, D., Beggs, A., Easteal, S., \& North, K. (2001). Differential expression of the actin-binding proteins, alpha-actinin-2 and -3 , in different species: implications for the evolution of functional redundancy. Human Molecular Genetics, 10(13), 1335-1346. doi:10.1093/hmg/10.13.1335

Moya, D., Madrigal, J., \& Zalasar, L. (2012). Angiotensin Converting Enzyme Insertion/Deletion Polymorphism and its Association with Complications in Patients with type 2 Diabetes Mellitus. Acta Médica Costarricense, 54(2), 102-108.

Muniesa, C., Santiago, C., Gómez-Gallego, F., \& Lucía, A. (2011). Genética y Deporte. Madrid: Consejo Superior de Deportes. Obtenido de https://sede.educacion.gob.es/ publiventa/genetica-y-deporte/ciencias-del-deporte/ 14554

NHGRI. (s.f). https://www.genome.gov. Obtenido de https:// www.genome.gov/es/genetics-glossary

Ozveren, Y., Ozcaldiran, B., Durmaz, B., \& Oral, O. (2014). Talent selection and genetics in sport. Turkish Journal of Sport and Exercise, 16(2), 1-8. doi:10.15314/ TJSE.201428098

Padullés i Riu, J. M., Terrados, N., Rodas, G., \& Campos, N. (2004). Genética y deporte. FORO J. M. CAGIGAL. Apunts. Educación física y deportes, 77, 85-87.

Papadimitriou, I., Papadopoulos, C., Kouvatsi, A., \& Triantaphyllidis, C. (2008). The ACTN3 gene in elite greek track and field athletes. International Journal of Sports Medicine, 29(4), 352-355. doi:10.1055/s-2007-965339

Pimenta, Coelho, Cruz, Morandi, Veneroso, Pussieldi, d. A., . .. Fernández, D. P. (2012). The ACTN3 genotype in soccer players in response to acute eccentric training. European Journal of Applied Physiology, 112(4), 1495-1503. doi:10.1007/s00421-011-2109-7

Pimenta, E., Coelho, D., Veneroso, C., Barros Coelho, E., Cruz, I., Morandi, R., . . . De Paz Fernández, J. (2013). Effect of ACTN3 Gene on Strength and Endurance in Soccer Players. Journal of Strength and Conditioning Research, 27(12), 3286-3292. doi:10.1519/ JSC.0b013e3182915e66

Puthucheary, Z., Skipworth, J., Rawal, J., Loosemore, M., Van Someren, K., \& Montgomery, H. (2011). The ACE Gene and Human Performance 12 Years On. Sports Medicine, 41(6), 433-448. doi:10.2165/11588720-000000000-00000

Rattigan, S., Dora, K., Tong, A., \& Clark, M. (1996). Perfused skeletal muscle contraction and metabolism improved by angiotensin II-mediated vasoconstriction. American Journal of Physiology, 271(1), 96-103. doi:10.1152/
ajpendo.1996.271.1.E96

Reilly, T., Bangsbo, J., \& Franks, A. (2000). Anthropometric and physiological predispositions for elite soccer. Journal of Sports Sciences, 18(9), 669-683. doi:10.1080/ 02640410050120050

Sánchez, J., Campuzano, Ó., Iglesias, A., \& Brugada, R. (2009). Genética y Deporte. Apunts Sports Medicine, 44(162), 86-97.

Santiago, C., Rodríguez Romo, Gómez Gallego, F., González Freire, M., Yvert, T., Verde, F., .. . Lucia, A. (2010). Is there an association between ACTN3 R577X polymorphism and muscle power phenotypes in young, non athletic adults? Scandinavian Journal of Medicine \& Science in Sports, 20(5), 771-778. doi:10.1111/j.16000838.2009.01017.x

Serra-Olivares, J., Prieto-Ayuso, A., Pastor-Vicedo, J., \& González-Víllora, S. (2020). Propuesta de evaluación multidisciplinar del talento de jóvenes futbolistas. Retos: Nuevas Tendencias en Educación Física, Deporte y Recreación, 38, 782-789.

Seto, J., Monkol, L., Quinlan, K., Houweling, P., Zheng, X., Garton, F., ... North, K. (2011). Deficiency of á-actinin-3 is associated with increased susceptibility to contractioninduced damage and skeletal muscle remodeling. Human Molecular Genetics, 20(15), 2914-2927. doi:10.1093/ hmg/ddr196

Sgourou, S., Fotopoulos, V., Kontos, V., Patrinos, G., \& Papachatzopoulou, A. (2012). Association of genome variations in the reninangiotensin system with physical performance. Human Genomics, 6(1), 6-24. doi:10.1186/ 1479-7364-6-24

Stølen, T., Chamari, K., Castagna, C., \& Wisløff, U. (2005). Physiology of Soccer. An Update. Sports Medicine, 35(6), 501-536. doi:10.2165/00007256-200535060-00004

Suraci, B., Quigley, C., Thelwell, R., \& Milligan, G. (2019). A Comparison of Training Modality and Total Genotype Scores to Enhance Sport-Specific Biomotor Abilities in Under 19 Male Soccer Players. The Journal of Strength \& Conditioning Research, [published online ahead of print, 2019 Nov 27]. doi:10.1519/JSC.0000000000003299

Ulucan, K., Sercan, K., \& Biyikli, T. (2015). Distribution of Angiotensin-1 Converting Enzyme Insertion/Deletion and á-Actinin-3 Codon 577 Polymorphisms in Turkish Male Soccer Players. Genetics \& Epigenetics, 7, 1- 4. doi:10.4137/GEGS31479

Unnithan, V., White, J., Georgiou, A., Iga, J., \& Drust, B. (2012). Talent identification in youth soccer. Journal of Sports Sciences, 30(15), 1719-1726. doi:10.1080/ 02640414.2012 .731515

Williams, A., \& Reilly, T. (2000). Talent identification and development in soccer. Journal of Sports Sciences, 18(9), 657-667. doi:10.1080/02640410050120041

Woods, D., Hickmann, M., Jamshidi, Y., Brull, D., Vassiliou, V., Jones, A., . . Montgomery, H. (2001). Elite swimmers and the D allele of the ACE I/D polymorphism. Human Genetics , 108(3), 230-232. doi:10.1007/s004390100466

Yang, N., MacArthur, D., Gulbin, J., Hahn, A., Beggs, A., Easteal, S., \& North, K. (2003). ACTN3 Genotype Is Associated with Human Elite Athletic Performance. American journal of human genetics, 73(3), 627-631. doi:10.1086/377590 